\title{
Cannabis and related products in the treatment of epilepsy
}

\author{
J. Helen Cross 1 \\ ${ }^{1}$ UCL Great Ormond Street Institute of Child Health, Great Ormond Street Hospital for Children NHS \\ Foundation Trust, London, and Young Epilepsy, Lingfield UK \\ Email Address: J. Helen Cross (h.cross@ucl.ac.uk) \\ Corresponding author: Professor JH Cross; Email: h.cross@ucl.ac.uk \\ DOI: https://doi.org/10.17724/jicna.2019.149
}

Received: 05 July 2018

Accepted: 30 May 2019

\begin{abstract}
There has been much heightened interest recently in the possible use of cannabinoid products in the treatment of complex epilepsies. Although systematic studies have now been performed and reported showing the benefit of cannabidiol (CBD) as Epidiolex (GW Pharma) in the treatment of Dravet and Lennox-Gastaut syndromes, there remains considerable confusion regarding the role of hemp oils and other products (CBD with tetrahydrocannabinol) for which there is no consistent, quality-assured product or evidence base for use. There is also a great deal of debate as to whether the psychoactive component, tetrahydrocannabinol (THC), is required for optimal effect, with no sound evidence base for support. The current position is that data has been acquired for Epidiolex, and a licence for use granted by the Federal Drug Administration, with data submitted to the European Medicines Authority. Other products with greater THC content have been reported to be beneficial although only in open-label studies, with results that could be considered little different to the randomised controlled trials with cannabidiol alone. Although benefit in certain specific complex epilepsies has been demonstrated with CBD, the requirement or not for some THC for added benefit remains under debate and as yet unproven.
\end{abstract}

Kewords: Cannabis, cannabinoids, cannabidiol, epilepsy, childhood.

(C) Cross JH.; licensee JICNA

\section{INTRODUCTION}

There has been much recent discussion with regard to the use of cannabis-related products in the treatment of epilepsy, with stories of effectiveness highlighted in the media, and the issue being discussed by the governments of Australia, the Netherlands and the United Kingdom. Heartwrenching stories and possible miracle cures are being reported widely. But what is the evidence?

\section{HISTORY}

Cannabis-related products have long been used in everyday life, as well as being used as recreational drugs. Merits have also long been claimed in the treatment of various medical conditions. In 2014, attention was brought to the potential utilisation of these products in the treatment of epilepsy through an internet report of a mother in Colorado who had researched the possible benefits - she had worked with a Colorado-based medical marijuana group to extract a cannabidiol-enriched oil that could be utilised for her daughter who had frequent seizures as a result of Dravet syndrome, a complex developmental epileptic encephalopathy with early onset. At the time of the trial she was experiencing nearly 50 convulsive seizures/day - by using the cannabidiol-rich product this decreased to two to three nocturnal convulsions/month [1] Subsequent social media and internet reports led to the belief that this must be a miracle treatment, specifically for Dravet syndrome.

Many parents therefore sought to utilise a similar extract, either producing it themselves, or sourcing it from producers in those states in the United States (US) where the product was legal, as well as other countries. As a result, Colorado became a significant source of the product, and a Facebook survey was subsequently generated, the results of which were published: 19 families reported, showing a positive effect against seizures in $>80 \%$, as well as improved sleep pattern, alertness and mood. Some negative effects were reported, not least in fatigue, drowsiness and loss of appetite [2]. While acknowledging the bias towards reporting about those who had experienced a positive effect, and the perception of cannabinoid products as 'natural', families have continued to seek what they see as the 'miracle' treat- 
ment.

\section{CANNABINOID PRODUCTS}

The two major neuroactive components in cannabis are the psychoactive compound D9 - tetrahydrocannabinol (THC) and the non-psychoactive cannabidiol (see figure 1).

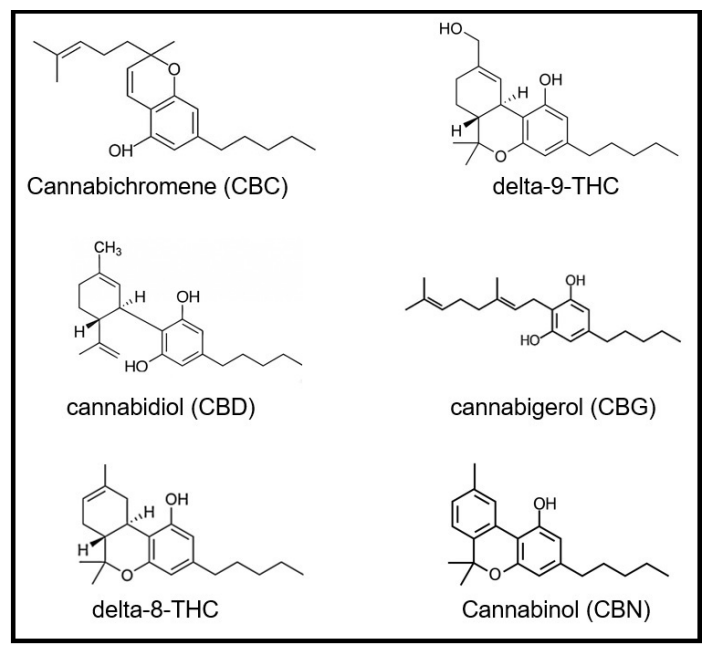

Figure 1 Structure of cannabinoids

The role of 'medical' marijuana has been much debated for the treatment of many different conditions. Individuals who use cannabis for recreational use have reported many benefits. However, the level of THC in the whole plant means that the drug has remained Schedule 1 in many countries, and is indeed illegal for any recreational or medical use without special licence. There is also great concern about utilising a product rich in THC within a specifically paediatric population; some in vitro studies have suggested THC can be proconvulsant, and follow-up studies evaluating users of cannabis have demonstrated limitation in performance in neuropsychological tests in those who start using the drug under the age of 15 years, compared to those starting at an older age [3]. The relative amount of THC in a product may determine whether it may be deemed 'legal' for purchase and utilisation. In the United Kingdom (UK), CBD extract containing $<0.2 \%$ THC has previously been considered legal. This would include the 'hemp oils' or CBD extract oils, available for purchase over the internet or from health food shops as nutritional products. These are however not produced to pharmaceutical standard. Studies have demonstrated that such products do not contain consistent amounts of the relative CBD and THC components [4], and there is therefore limited reliability despite the product information given. No analytical controls are mandatory, there is no legal protection or guarantee about the composition and quality, and there is no obligatory testing or basic regulatory framework to determine indication area, daily dosage, route of administration, maximum recommended daily dose, packaging, shelf life, or stability. With fluctuations in the relative components of the products, and no information in dosing, it may not be regarded as safe practice to prescribe or endorse such products for utilisation. The Medicines and Healthcare Products Regulatory Agency in the UK examined this issue and released a position statement in 2016 'products containing cannabidiol (CBD) used for medical purposes are a medicine. Medicinal products must have a product licence (marketing authorisation) before they can be legally sold, supplied or advertised in the UK, unless exempt. Licensed medicinal products have to meet safety, quality and efficacy standards to protect public health' (https://www.gov.uk/government/news/ mhra-statement-on-products-containing-cannabidiol-cbd). Aside from the policing of such use, this proved impossible.

A range of cannabidiol-containing products have been utilised for different medical conditions in countries where THC content is not restricted. These are galenical oils prepared by pharmacists following medical prescription legal in several European countries such as the Netherlands, Italy and Germany. Such products may be prescribed by registered physicians. Dutch-produced Bedrocan varieties are frequently used for the preparation of galenic CBDbased oils. More recently, Epidiolex, a liquid formulation of pure plant-derived cannabidiol, or CBD, <0.1\% THC (GW Pharma), has been evaluated for treating a number of rare childhood-onset epilepsy disorders. It is produced in a consistent quality-assured way to pharmaceutical standard, so there is a reliability to the content and stability. It is registered as a pharmaceutical, to be assessed in appropriate clinical trials, and data is to be submitted to the appropriate regulatory authorities for licence. The FDA approved a licence for the product for use in certain complex childhood epilepsies in June 2018; the European Medicines Authority (EMA) are currently assessing the medication and are due to report early 2019.

More recently, after full review, legislation in the UK has been changed so that physicians can prescribe cannabisbased medicines when it has been agreed that their patients could benefit from this treatment, with further guidance for children with epilepsy provided by the British Paediatric Neurology Association (https://bpna.org.uk/userfiles/ BPNA_CBPM_Guidance_Oct2018.pdf), while stating that Epidiolex is the only product for which there is evidence and a safety base, and evidence with regard to prescribing information.

\section{CANNABINOIDS AND EPILEPSY}

\section{SCIENTIFIC DATA}




\section{PRECLINICAL TRIALS}

Despite the long history of the use of cannabis-related products, the endocannabinoid system was not described until the 1980s/90s. Cannabidiol was isolated as early as 1940 , and its structure elucidated in 1963, with THC isolated in 1964. The ensuing interest focused on the psychoactive component of this compound. The anticonvulsant effects of CBD, THC and other cannabinoids have been compared using a variety of standard seizure models [5]Despite the long history of the use of cannabis-related products, the endocannabinoid system was not described until the 1980s/90s. Cannabidiol was isolated as early as 1940, and its structure elucidated in 1963, with THC isolated in 1964. The ensuing interest focused on the psychoactive component of this compound. The anticonvulsant effects of CBD, THC and other cannabinoids have been compared using a variety of standard seizure models $[6,7]$.

Cannabidiol is the only non-THC phytocannabinoid to have been assessed in preclinical and clinical studies for anticonvulsant effects. The anticonvulsant profile of CBD has specifically been demonstrated in acute seizure models, but not so convincingly in animal models of chronic epilepsy [8]. Cannabidivarin (CBDV), the propyl variant of CBD, also has significant anticonvulsant properties, with and without concomitant anticonvulsant medications (sodium valproate, ethosuximide, phenobarbitone) [6]. CBDV exerts its effects via the $\mathrm{CB} 1$ receptor independent mechanism [9]. CBD and related compounds may reduce neuronal excitability and neuronal activity, but the exact mechanism through which they exert an anti-epileptic effect is not currently known.

\section{CLINICAL DATA}

Clinical use of cannabis-related products in epilepsy has been scant until recently. A Cochrane review published in 2014 reported four randomised trial reports where cannabidiol was the treatment agent [10]. There was however only a total of 48 patients and all four studies were methodologically flawed. Details of randomisation were not included in any study report. There was no investigation of whether the control and treatment participant groups were the same or different. All four reports were low quality, and only dealt with the secondary outcome of adverse effects. None of the patients in the treatment groups suffered adverse effects. The authors did not think that any reliable conclusions could be drawn regarding the efficacy of cannabinoids as a treatment for epilepsy. Cannabidiol had been safely administered to small numbers of patients generally for short periods of time, and so the safety of longterm cannabidiol treatment could not be reliably assessed.

A more recent systematic review determined 35 publications from 36 studies that met inclusion criteria with a further 10 studies yet to be published [11]. Studies were included if they administered plant-based and pharmaceutical cannabinoids to prevent or treat epilepsy and epileptic seizures in participants of any age, with any type of epilepsy or seizure. All experimental and epidemiological study de- signs were included, including randomised controlled trials (RCTs), non-RCTs, quasi-experimental, before and after studies, prospective and retrospective cohort studies, casecontrol studies, analytical cross-sectional studies, self-report surveys and case reports. This included six randomised controlled trials; the three outlined in the previous Cochrane review, and three new RCTs reporting on utilisation of Epidiolex as produced by GW Pharma in Dravet syndrome and Lennox Gastaut syndrome (LGS). Of the 30 observational studies, six were open-label intervention trials, 10 were case studies, eight were self-report surveys, five were retrospective chart reviews, and in one the design of the remaining study was unclear.

\section{CANNABIDIOL IN EPILEPSY}

Epidiolex (CBD $<0.1 \%$ THC, GW Pharma) has been evaluated in clinical trials in the treatment of complex epilepsies. Initially, approval from the FDA was achieved for its utilisation as an investigational new drug in the open-label treatment of children and adults with complex epilepsy across six centres in the USA [12]. To qualify for inclusion, individuals had to have intractable early onset epilepsy, be on more than three AEDs, not including a ketogenic diet or vagal nerve stimulation, have a non-progressive disorder, and have no significant abnormalities on laboratory testing. They would be requested to keep a four-week baseline seizure diary, would then be initiated on cannabidiol (CBD) $5 \mathrm{mg} / \mathrm{kg} /$ day, titrated at $2-5 \mathrm{mg} / \mathrm{kg} /$ day increments until tolerance or a maximum of $25 \mathrm{mg} / \mathrm{kg} /$ day. Laboratory investigations were evaluated at four, eight and 12 weeks. Two hundred and fourteen patients were enrolled, with 167 completing for safety and tolerability analysis, and 137 for efficacy analysis. Thirty-nine percent experienced $>50 \%$ seizure reduction over a 12-week period, with 15 seizure-free for the last four weeks, and five seizure-free for the whole treatment period [12]. Further analysis of those with Dravet syndrome and LGS showed $49 \%$ and $37 \%$ responders respectively. Adverse events were seen in $79 \%$, most commonly somnolence (25\%), decreased appetite and diarrhoea (each 19\%). The overall conclusion of the study was that CBD might reduce seizure frequency, and might have an adequate safety profile in children and young adults with highly treatment-resistant epilepsy. However, it became clear that through an effect on cytochrome p450 system in the liver, the norclobazam metabolite of clobazam was dramatically increased when patients were on concomitant treatment [13]. This was separately reported prior to the results of the full study, where it was also clear that many of the side-effects reported from the CBD could be attributed to increased clobazam metabolite levels. Further analysis in the open-label study revealed that $36 / 70$ patients $(51 \%)$ on clobazam experienced more than a $50 \%$ reduction of seizures, compared to $18 / 67$ (27\%) not on clobazam. On multiple logistic regression, clobazam use was the only independent predictor of a $>50 \%$ reduction in motor seizures. 
Subsequent randomised controlled trials of CBD vs placebo as an add-on treatment of Dravet and LGS have been published [14, 15, 16]. For the Dravet syndrome study, children with a definitive diagnosis of Dravet syndrome (as assessed by clinical criteria via the Epilepsy Study Consortium), were recruited from 23 centres across the USA and Europe. A total of 177 children aged two to 18 years were screened, with 120 ultimately randomised to either CBD (up to $20 \mathrm{mg} / \mathrm{kg}$ /day) or placebo, having experienced at least four convulsive seizures over the four-week baseline period. The mean age in the CBD group (9.7 years) was not different from the placebo group (9.8 years). The median number of anti-epileptic drugs trialled was four, and the patients were taking a median of three (range 1-5), most commonly clobazam (65\%), valproate (59\%), stiripentol (42\%), levetiracetam (28\%) and topiramate (26\%). In the CBD group, the primary endpoint of convulsive seizure frequency at the end of the treatment period decreased by a median $-38.9 \%$ from the baseline. This was significantly greater than in the placebo group $(-13.3 \%, \mathrm{p}=0.01))$. The responder rate ( $>50 \%$ reduction in seizures) was $43 \%$ in the CBD group vs $27 \%$ in the placebo group; this did not reach significance $(\mathrm{p}=0.08)$. Adverse events during the treatment period were reported in $93 \%$ CBD vs $75 \%$ placebo; $89 \%$ adverse events were mild or moderate. In both groups, the first occurrence of an adverse event was most commonly reported during the 14 days dose-escalation. Common adverse events ( $>10 \%$ frequency) in the CBD group were vomiting, fatigue, pyrexia, upper respiratory tract infection, decreased appetite, convulsion, lethargy, somnolence and diarrhoea. In the CBD group, eight patients withdrew from the trial owing to adverse events, as compared with one in the placebo group.

In this study, $65 \%$ of children with Dravet syndrome were taking clobazam. As highlighted above, there is no question that norclobazam levels rise when CBD and clobazam are given together, and some of the side-effects, such as somnolence, could well be attributed to this. Some may question whether any of the CBD effects on seizures is the result of such an interaction, or a direct effect of the medication itself. Prior to the Dravet study, a pharmacokinetic dose-finding study was performed and recently published [17]. Thirty-four children with Dravet syndrome aged four to 10 years were randomised $4: 1$ to $\operatorname{CBD}(5,10$ or $20 \mathrm{mg} / \mathrm{kg} /$ day) or placebo taken twice a day; 32 completed treatment. The double-blind trial comprised a four-week baseline, three-week treatment, 10-day taper and four-week follow-up. Pharmacokinetic sampling for the measurement of CBD, metabolites and anti-epileptic drug levels were performed on the first day of dosing and at the end of treatment. CBD did not effect concomitant AED levels, except for norclobazam. Norclobazam levels increased on all doses of CBD, but not placebo, and not if there was concomitant stiripentol. Although numbers were small with only four patients taking both clobazam and stiripentol, this suggests that stiripentol maximally inhibits CYP2C19. In the Dravet trial, $65 \%$ of the children were also taking clobazam, and
$42 \%$ stiripentol [15]. Although in those where stiripentol was taken concomitantly, clobazam metabolites were unlikely to have risen further. Subgroup analysis was not possible owing to small numbers [18].

With regard to LGS, two separate studies of similar methodology have been published. In the initial publication, the study was across 24 sites in the USA, the Netherlands and Poland [14]. One hundred and seventy-one patients with LGS, as verified through criteria by the Epilepsy Consortium, were included. Patients who were refractory (i.e., inadequately managed on at least two anti-epileptic drugs, inclusive of previous and current treatments), were taking one to four anti-epileptic drugs, and who had at least two drop seizures per week during the four-week baseline period, were eligible. After a four-week screening period, patients were randomised to receive $\mathrm{CBD}(\mathrm{N}=86)$, increased to a dose of $20 \mathrm{mg} / \mathrm{kg} /$ day, or placebo $(\mathrm{N}=85)$, and received treatment for 14 weeks. The primary endpoint was mean percentage reduction in drop seizures frequency from baseline. In the CBD group, the monthly frequency of drop seizures decreased by a median of $43.9 \%$ from baseline over the 14 -week treatment period compared to $21.8 \%$ in the placebo group; a difference that reached statistical significance. There was also a significant difference between groups in the reduction of total seizures. With regard to adverse events, all-cause adverse events were reported in $86 \%$ of the CBD group and $69 \%$ of the placebo group. Those occurring in $>10 \%$ in the CBD group included somnolence and diarrhoea. These events led to withdrawal in 12/86 of the CBD group and 1/85 of the placebo group. In the second publication, two different doses of CBD were examined, compared to placebo [16]. A total of 225 patients aged two to 55 years were enrolled with confirmed LGS as per clinical criteria, from 30 clinical centres, and randomised to placebo, $10 \mathrm{mg} / \mathrm{kg} / \mathrm{day}$ CBD or $20 \mathrm{mg} / \mathrm{kg} /$ day. The median percent reduction from baseline in drop-seizure frequency during the treatment period was $41.9 \%$ in the $20 \mathrm{mg}$ CBD group, $37.2 \%$ in the $10 \mathrm{mg}$ CBD group, and $17.2 \%$ in the placebo group ( $\mathrm{P}=0.005$ for the $20 \mathrm{mg}$ CBD group vs placebo group, and $\mathrm{P}=0.002$ for the $10 \mathrm{mg}$ CBD group vs placebo group). Adverse events were reported in 77 of 82 patients (94\%) in the 20mg CBD group, in 56 of 67 patients (84\%) in the 10mg CBD group, and in 55 of 76 patients $(72 \%)$ in the placebo group. The most common adverse events among the patients in the CBD groups were somnolence, decreased appetite, and diarrhoea; these events occurred more frequently in the higher-dose group. Six patients in the 20mg CBD group and one patient in the 10mg CBD group discontinued the trial medication because of adverse events and were withdrawn from the trial. Elevation of serum aminotransferase concentrations was the most common adverse event among these patients, occurring in four of the six patients in the $20 \mathrm{mg} / \mathrm{kg}$ CBD group as well as in the patient in the $10 \mathrm{mg} / \mathrm{kg}$ CBD group, with maximum elevations in aspartate aminotransferase or alanine aminotransferase concentrations that were 3.2 to 12.2 times the upper limit of the normal range. Further publi- 
cations have reported on observational studies in a wider range of complex epilepsies, as well as extended follow-up of the expanded access programme. Observational studies have suggested efficacy in epilepsy associated with CDKL5 disorder, Aicardi syndrome, Doose syndrome and 15q deletion syndrome [19], as well as Febrile Infection Related Epilepsy Syndrome (FIRES) [20], epilepsy associated with tuberous sclerosis[21] and epilepsy associated with Sturge Weber syndrome [22]. These data suggest wider efficacy across a range of complex epilepsies beyond the selected groups for the RCTS. Expansion of the open access programme with follow-up up to 96 weeks has shown sustained efficacy in a wider range of complex epilepsies with $76 \%$ retention; treatment-emergent adverse events were similar to previous reports with convulsions (9\%), status epilepticus $(7 \%)$, pneumonia $(5 \%)$, and vomiting $(3 \%)$ the most frequently reported [23].

One question remains as to whether seizure outcome is the only benefit that may be seen utilising cannabinoid products. Trials are being undertaken in children with developmental disorders, but without epilepsy. Further in the Epidiolex trials, a secondary outcome was reporting on any change seen in the Caregiver Global Impression of Change (CGIC) scale, assessed on a seven-point Likert-like scale that used three categories of improvement (slightly improved, much improved, or very much improved), three categories of worsening (slightly worse, much worse, or very much worse), and an option of 'no change'. In all studies, significantly more parents reported an improvement in the scale in the CBD group as compared to the placebo group $[14,15,16]$. In a further reported subset of patients enrolled in a prospective, open-label clinical study where they analysed the caregiver-reported Quality of Life in Childhood Epilepsy (QOLCE), results from caregivers of 48 patients indicated an 8.2 \pm 9.9-point improvement in overall patient QOLCE ( $\mathrm{p}<0.001)$ following 12 weeks of CBD. Subscores with improvement included energy/fatigue, memory, control/helplessness, other cognitive functions, social interactions, behaviour, and global QOL, differences that were not correlated to changes in seizure frequency or adverse events [24]. These findings suggest that effects beyond seizure outcome warrant further evaluation.

\section{STUDIES UTILISING ALTERNATIVE PRODUCTS}

In the recently published systematic review by Stockings et $\mathrm{al}[11]$, by far the majority of publications reviewed were observational and open-label. A preliminary report on an RCT using a transdermal CBD gel (Zynerba) in refractory focal epilepsy in adults showed a statistical reduction in seizures with either of two doses compared to placebo over a 12-week period, although with meaningful clinical reductions in seizures in the open-label extension (https://zynerba.com/zynerba-pharmaceuticalsannounces-twelve-month-zyn002-data-from-star-2-study- in-patients-with-focal-seizures-at-the-2018-annualmeeting-of-the-american-academy-of-neurology-aan/). The question remains open however as to whether the full benefit is obtained from pure CBD, or whether additional benefit may be gained by combining it with THC to some extent. A recent case series from Israel reported on the experience of five paediatric epilepsy clinics utilising CBD-enriched medical cannabis with 1\% THC [25]. They reported on 74 patients who had previously failed on up to seven anti-epileptic drug treatments, some of which included a ketogenic diet and vagal nerve stimulation. Although they reported a positive effect, only $18 \%$ reported $>75 \%$ reduction in seizures, and $34 \%$ reported a $50-75 \%$ reduction. Although this was an open-label study, these figures appear little different to that seen in the RCTs of CBD alone. Similar adverse effects were also reported including somnolence, fatigue, gastrointestinal disturbance and irritability, leading to discontinuation in five patients. A further study utilising CBD with $2 \%$ THC from Canada reported on open-label use in 20 children with Dravet syndrome. The dose ranged from $2-6 \mathrm{mg} / \mathrm{kg} /$ day of CBD and $0.04-0.32 \mathrm{mg} / \mathrm{kg} /$ day of THC. Nineteen patients completed 20 weeks intervention; one child who died of SUDEP was excluded from the analysis. As with the RCTs, somnolence, anorexia and diarrhoea were the most common adverse events seen, with abnormalities of liver transaminases and platelets observed with concomitant valproic acid therapy. Following 20 weeks therapy, $12 / 20$ reported $>50 \%$ reduction of seizures [26], again not dissimilar figures to the RCTs of CBD.

\section{CURRENT POSITION}

There is much confusion about different cannabinoidrelated products and their proven role (or not) in the treatment of epilepsy. Parents and families are often desperate to leave no stone unturned in the treatment of devastating conditions. News travels fast via the internet and should a possible effective treatment be reported, families are understandably keen to pursue this. Further, a product derived from a plant may be perceived to be 'natural', particularly if available as a 'health' food. As highlighted above, however, only the pharmaceutically derived product Epidiolex has been trialled in a systematic way for efficacy and safety. Hemp oils are not produced in a similar quality-assured way, and content is not consistent. Further, there is no reliable evidence to indicate an appropriate dose. When children do not respond to a product, however, there is speculation thereafter as to why this may not be the case. The question arises as to whether THC content may be required, and consequently tried. Again there is no evidence to support this; one also has to question the safety of such a suggestion while being aware of this as the psychoactive component, although further research is warranted. Parental reporting studies can also be subject to bias; a recent study enrolled 75 patients of which $57 \%$ reported no improvement in seizure 
control, whereas $33 \%$ reported a $50 \%$ reduction in seizures. There was however a marked differential in reporting according to whether a family resided in Colorado prior to starting CBD, or moved there in order to acquire the product - for those already residing in Colorado the responder rate was $22 \%$ vs $47 \%$ of those who moved there to acquire the preparation [27].

The data with regard to Epidiolex has been acquired in the standard regulatory format, and data submitted to the regulatory bodies for consideration of licence as an orphan medication in the treatment of LGS and Dravet syndrome. Efficacy studies appear promising, highlighting the medication to be effective, as with any other standard anti-epileptic drug in a complex population highly resistant to other medications. Further, although there was initially some debate as to the possible effect being the result of drug interaction with clobazam, the LGS population are less likely to have this as a co-medication, and pharmacokinetic studies suggest an independent effect. Further studies are ongoing in infantile spasms and epilepsy associated with tuberous sclerosis.

\section{CONCLUSION}

As a medical profession we need to consider code of conduct. Although we appreciate that our treatment options in these devastating syndromes are limited, we have no evidence that oils with or without THC offer anything over and above standard pharmaceutical CBD, for which appropriate evidence base has now been acquired. Our responsibility lies with the safety of our patients; first and foremost we should do no harm.

This is an Open Access article distributed under the terms of the Creative Commons Attribution License (http://creativecommons.org/licenses/by/4.0), which permits unrestricted use, distribution, and reproduction in any medium, provided the original work is properly credited. The Creative Commons Public Domain Dedication waiver (http://creativecommons.org/publicdomain/zero/1.0/) applies to the data made available in this article, unless otherwise stated.

\section{Cite this article as:}

Cross, J. H. (2019). Cannabis and related products in the treatment of epilepsy. Journal of the International Child Neurology Association, 1(1). https://doi.org/10.17724/ jicna.2019.149

\section{References}

[1] Maa E, Figi P. The case for medical marijuana in epilepsy. Epilepsia. 2014;55(6):783-786. PubMed.

[2] Porter BE, Jacobson C. Report of a parent survey of cannabidiol-enriched cannabis use in pediatric treatment-resistant epilepsy. Epilepsy \& Behavior. 2013 Dec;29(3):574-577. PubMed.

[3] Fontes MA, Bolla KI, Cunha PJ, Almeida PP, Jungerman F, Laranjeira RR, et al. Cannabis use before age 15 and subsequent executive functioning. The British journal of psychiatry : the journal of mental science. 2011 Jun;198(6):442-7. PubMed.

[4] Vandrey R, Raber JC, Raber ME, Douglass B, Miller C, Bonn-Miller MO. Cannabinoid Dose and Label Accuracy in Edible Medical Cannabis Products. JAMA. 2015 06;313(24):2491-2493. PubMed.

[5] KARLER R, TURKANIS SA. CANNABIS AND EPILEPSY. In: NAHAS GG, PATON WDM, editors. Marihuana Biological Effects. Pergamon; 1979. p. 619 - 641. PubMed.

[6] Jones NA, Hill AJ, Smith I, Bevan SA, Williams CM, Whalley BJ, et al. Cannabidiol displays antiepileptiform and antiseizure properties in vitro and in vivo. The Journal of pharmacology and experimental therapeutics. 2010 Feb;332(2):569-577. PubMed.

[7] Jones NA, Glyn SE, Akiyama S, Hill TDM, Hill AJ, Weston SE, et al. Cannabidiol exerts anti-convulsant effects in animal models of temporal lobe and partial seizures. Seizure - European Journal of Epilepsy. 2012 Jun;21(5):344-352. PubMed.

[8] Colasanti BK, Lindamood C, Craig CR. Effects of marihuana cannabinoids on seizure activity in cobaltepileptic rats. Pharmacology Biochemistry and Behavior. 1982;16(4):573 - 578. PubMed.

[9] Hill TDM, Cascio MG, Romano B, Duncan M, Pertwee RG, Williams CM, et al. Cannabidivarin-rich cannabis extracts are anticonvulsant in mouse and rat via a CB1 receptor-independent mechanism. British Journal of Pharmacology. 2013;170(3):679-692. PubMed.

[10] Gloss D, Vickrey B. Cannabinoids for epilepsy. Cochrane Database of Systematic Reviews. 2014;(3). PubMed.

[11] Stockings E, Zagic D, Campbell G, Weier M, Hall WD, Nielsen S, et al. Evidence for cannabis and cannabinoids for epilepsy: a systematic review of controlled and observational evidence. Journal of Neurology, Neurosurgery \& Psychiatry. 2018;89(7):741-753. PubMed.

[12] Devinsky O, Marsh E, Friedman D, Thiele E, Laux L, Sullivan J, et al. Cannabidiol in patients with treatment-resistant epilepsy: an open-label interventional trial. The Lancet Neurology. 2016 Mar;15(3):270-278. PubMed. 
[13] Geffrey AL, Pollack SF, Bruno PL, Thiele EA. Drug-drug interaction between clobazam and cannabidiol in children with refractory epilepsy. Epilepsia. 2015;56(8):1246-1251. PubMed.

[14] Thiele EA, Marsh ED, French JA, MazurkiewiczBeldzinska M, Benbadis SR, Joshi C, et al. Cannabidiol in patients with seizures associated with LennoxGastaut syndrome (GWPCARE4): a randomised, double-blind, placebo-controlled phase 3 trial. The Lancet. 2018 Mar;391(10125):1085-1096. PubMed.

[15] Devinsky O, Cross JH, Laux L, Marsh E, Miller I, Nabbout R, et al. Trial of Cannabidiol for Drug-Resistant Seizures in the Dravet Syndrome. New England Journal of Medicine. 2017;376(21):2011-2020. PubMed.

[16] Devinsky O, Patel AD, Cross JH, Villanueva V, Wirrell EC, Privitera M, et al. Effect of Cannabidiol on Drop Seizures in the Lennox-Gastaut Syndrome. New England Journal of Medicine. 2018;378(20):1888-1897. PubMed.

[17] Devinsky O, Patel AD, Thiele EA, Wong MH, Appleton R, Harden CL, et al. Randomized, dose-ranging safety trial of cannabidiol in Dravet syndrome. Neurology. 2018;90(14):e1204-e1211. PubMed.

[18] Devinsky O, Cross JH, Laux L, Marsh E, Miller I, Nabbout $\mathrm{R}$, et al. Trial of Cannabidiol for Drug-Resistant Seizures in the Dravet Syndrome. New England Journal of Medicine. 2017;376(21):2011-2020. PubMed.

[19] Open-label use of highly purified CBD (Epidiolex $\AA$ ) in patients with CDKL5 deficiency disorder and Aicardi, Dup15q, and Doose syndromes. Epilepsy \& Behavior. 2018;86:131 - 137. PubMed.

[20] Gofshteyn JS, Wilfong A, Devinsky O, Bluvstein J, Charuta J, Ciliberto MA, et al. Cannabidiol as a Potential Treatment for Febrile Infection-Related Epilepsy Syndrome (FIRES) in the Acute and Chronic Phases. Journal of Child Neurology. 2017;32(1):3540. PubMed.

[21] Hess EJ, Moody KA, Geffrey AL, Pollack SF, Skirvin LA, Bruno PL, et al. Cannabidiol as a new treatment for drug-resistant epilepsy in tuberous sclerosis complex. Epilepsia. 2016;57(10):1617-1624. PubMed.

[22] Kaplan EH, Offermann EA, Sievers JW, Comi AM. Cannabidiol Treatment for Refractory Seizures in Sturge-Weber Syndrome. Pediatric Neurology. 2017;71:18 - 23.e2. PubMed.

[23] Szaflarski JP, Bebin EM, Comi AM, Patel AD, Joshi C, Checketts D, et al. Long-term safety and treatment effects of cannabidiol in children and adults with treatment-resistant epilepsies: Expanded access program results. Epilepsia. 2018;59(8):1540-1548. PubMed.
[24] Rosenberg EC, Louik J, Conway E, Devinsky O, Friedman D. Quality of Life in Childhood Epilepsy in pediatric patients enrolled in a prospective, openlabel clinical study with cannabidiol. Epilepsia. 2017;58(8):e96-e100. PubMed.

[25] Tzadok M, Uliel-Siboni S, Linder I, Kramer U, Epstein O, Menascu S, et al. CBD-enriched medical cannabis for intractable pediatric epilepsy: The current Israeli experience. Seizure. 2016;35:41 - 44. PubMed.

[26] McCoy B, Wang L, Zak M, Al-Mehmadi S, Kabir $\mathrm{N}$, Alhadid $\mathrm{K}$, et al. A prospective open-label trial of a CBD/THC cannabis oil in dravet syndrome. Annals of Clinical and Translational Neurology. 2018;5(9):1077-1088. PubMed.

[27] Press CA, Knupp KG, Chapman KE. Parental reporting of response to oral cannabis extracts for treatment of refractory epilepsy. Epilepsy \& Behavior. 2015;45:49 52. PubMed. 